\title{
A novel approach to negative feedback in RX front-ends
}

\author{
Vandi, Luca; Andreani, Pietro; Tired, Tobias; Mattisson, Sven
}

Published in:

Proceedings of IEEE 24th Norchip Conference

Link to article, DOI:

10.1109/NORCHP.2006.329217

Publication date:

2006

Document Version

Publisher's PDF, also known as Version of record

Link back to DTU Orbit

Citation (APA):

Vandi, L., Andreani, P., Tired, T., \& Mattisson, S. (2006). A novel approach to negative feedback in RX frontends. In Proceedings of IEEE 24th Norchip Conference (Vol. 1, pp. 231-234). IEEE.

https://doi.org/10.1109/NORCHP.2006.329217

\section{General rights}

Copyright and moral rights for the publications made accessible in the public portal are retained by the authors and/or other copyright owners and it is a condition of accessing publications that users recognise and abide by the legal requirements associated with these rights.

- Users may download and print one copy of any publication from the public portal for the purpose of private study or research.

- You may not further distribute the material or use it for any profit-making activity or commercial gain

- You may freely distribute the URL identifying the publication in the public portal

If you believe that this document breaches copyright please contact us providing details, and we will remove access to the work immediately and investigate your claim 


\title{
A novel approach to negative feedback in $\mathrm{RX}$ front-ends
}

\author{
Luca Vandi $^{(1)}$, Pietro Andreani ${ }^{(1)}$, Tobias Tired ${ }^{(2)}$ and Sven Mattisson ${ }^{(2)}$ \\ (1) Ørsted-DTU, Centre for Physical Electronics, Ørsteds Plads, DK-2800 Kgs. Lyngby, Denmark. \\ ${ }^{(2)}$ Ericsson AB, Nya Vattentornet, SE-22183 Lund, Sweden. \\ lv@oersted.dtu.dk
}

\begin{abstract}
A new approach to negative feedback is proposed and applied to active mixer cells based on Gilbert multiplier. The feedback can be exploited in several ways, and different configurations are derived. A dual-loop topology provides a solution for inductor-less broad-band receiver stages. The nature of the dual loop ensures controlled gain and input matching, and a first-order linearity analysis is presented. A single-loop narrow-band topology is derived from more traditional implementations; main characteristics are examined thoroughly, with specific respect to input matching, noise and stability issues.
\end{abstract}

\section{Introduction}

Traditional active mixers based on the Gilbert cell [1], like the one depicted in Figure 1(a), are ubiquitous in modern RF architectures. The design of such cells is carried out under the general assumption that the linearity of the block is intimately related to the linearity of the transconductor ( $M_{1}$ in the figure). To improve linearity performances, several solutions have been devised, most of which are based on a combination of the following methods [2]: feedback, feedforward, predistortion and piecewise approximation. Feedback-based solutions have been traditionally relegated to low-frequency applications, because of the intrinsic bandwidth reduction associated with the feedback. As more advanced technologies become available, bandwidth limitations become less and less critical. To fully exploit the capabilities of negative feedback, it would be beneficial to embrace the complete cell (transconductor and switching pair) into the feedback loop, rather than simply closing the loop locally by means of source degeneration as illustrated in Figure 1(b). A solution is sketched in Section 2, and is further developed in Section 3, where a complete dual-loop wide-band receiver front-end is proposed. In the design of a receiver chain, linearity and noise are closely related; a narrow-band solution is proposed in Section 4 , where the same principle described in the following is applied in order to improve noise performances. Conclusions are drawn in Section 5.

\section{Basic concept}

The cell in Figure 1(c) is perfectly equivalent to the one in Figure 1(a) with respect to the downconverted signal. The node $v_{m}$, however, has some interesting properties at high frequency. We model the differential pair $\left(M_{2}-M_{3}\right)$ as a couple of switches, controlled by a time-varying periodic function at frequency $f_{L O}$, like in Figure 1(d), indicating the function as $m_{L O}(t)$. When passives are connected like in Figure 1(c), the computation of the output voltages is more involved, and we will develop it with the aid of Figure 2 . We can easily show that the following relations hold:

$$
\begin{gathered}
v_{h}=i_{i n} R \frac{1+s \frac{C R}{2}}{1+s C R} \quad ; \quad v_{t}=i_{i n} \frac{R}{2} \frac{s C R}{1+s C R} \\
v_{m}=i_{i n} \frac{R}{2} .
\end{gathered}
$$

The application to the structure in Figure 1(c) is straigthforward, due to the symmetry properties:

$$
\begin{aligned}
v_{I F}= & -i_{R F} \frac{1+m_{L O}}{2} \frac{v_{h}}{i_{i n}}-i_{R F} \frac{1-m_{L O}}{2} \frac{v_{t}}{i_{i n}}- \\
& -\left(-i_{R F} \frac{1+m_{L O}}{2} \frac{v_{t}}{i_{i n}}-i_{R F} \frac{1-m_{L O}}{2} \frac{v_{h}}{i_{i n}}\right) \\
= & -i_{R F} \cdot m_{L O}\left(\frac{v_{h}}{i_{i n}}-\frac{v_{t}}{i_{i n}}\right) \\
= & -i_{R F} \cdot m_{L O} \cdot \frac{R}{1+s C R}, \\
v_{m}= & -i_{R F} \frac{1+m_{L O}}{2} \frac{R}{2}-i_{R F} \frac{1-m_{L O}}{2} \frac{R}{2} \\
= & -i_{R F} \frac{R}{2} .
\end{aligned}
$$

As we can see in (3) the mixing properties in downconvertion are exactly as expected. Furthermore, the input RF current is amplified at node $v_{m}$ without any kind of filtering nor mixing. This node provides a replica of the RF input current $i_{R F}$, and it will be fed back into the loop in order to linearize the mixer's behaviour.

\section{Dual-loop wide-band implementation}

Traditional single-stage broad-band LNAs for RF applications present a fundamental trade-off between input matching, noise figure and linearity. This trade-off might turn out to be simply not good enough for the particular application: several solutions have been proposed, among which feed-forward [3] and negative feedback should be mentioned. When linearity is a serious concern, negative 


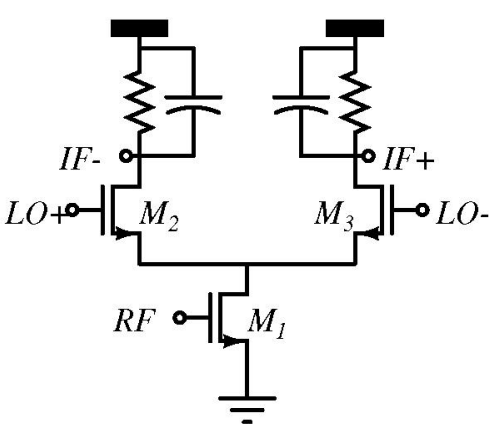

(a)

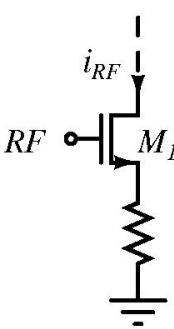

(b)

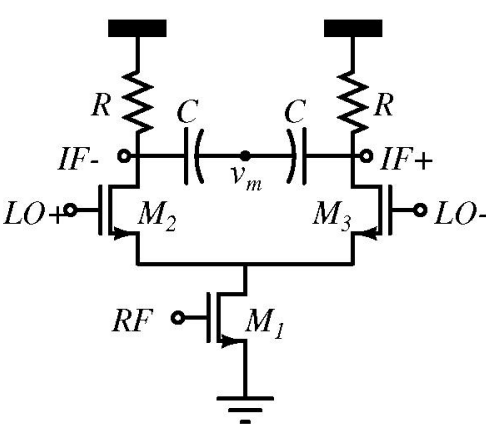

(c)

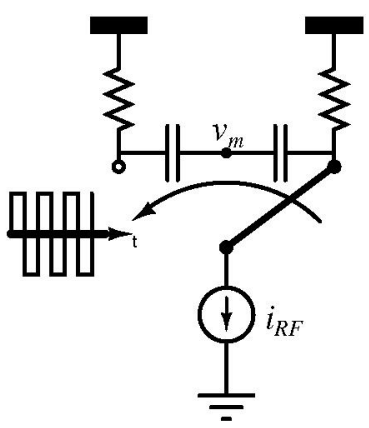

(d)

Figure 1: Down-conversion mixers based on the Gilbert cell: typical configuration (a), source-degeneration (b), proposed approach (c) and full-switching model (d).

feedback is the preferred choice, but certain limitations occur when a single-loop feedback is implemented. As an example, we can examine the symplified schematic in Figure (3), from which it is easy to get the following result:

$$
Z_{\text {in }}=\frac{R_{f}}{1+A}
$$

When we calculate the loop gain we have to impose the input-matching condition $Z_{i n}=R_{S}$, and as a result we get:

$$
G_{\text {loop }}=-\frac{A}{A+2} ; G \triangleq \frac{v_{o u t}}{v_{S}}=-\frac{A}{2}
$$

We can notice that the loop-gain magnitude $\left|G_{\text {loop }}\right|$ is even smaller than one. This solution is not very robust, because the forward gain $-A$ must be accurately controlled in order to provide the correct input matching, but no feedback can be exploited to provide such accuracy. Moreover, nonlinearities in the gain stage get no attenuation whatsoever. For all these reasons, a dual-loop solution is a more sensible choice. The proposed approach is depicted in Figure 4(a); to simplify the analysis of the stage, we consider only the behaviour at high frequency. Assuming that the impedance of low-pass capacitors $C$ is negligible, the equivalent resistance at node $v_{m}$ is $R_{m i x}=\frac{R}{2}$. We can define $R_{1}=\frac{R}{2} / / R_{s 1}$ and the equivalent circuit looks as in Figure 4(b): the output signal is actually $i_{2}$, which is the RF current that is going to be down-mixed and converted into a differential voltage signal. In this simplified picture, the circuit operates at a single frequency. From the figure

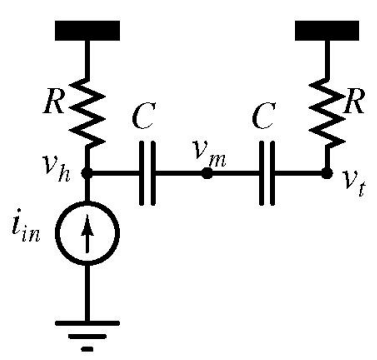

Figure 2: R-C low-pass network . we can derive a proper set of equations as follows:

$$
\begin{aligned}
v_{a}=v_{S}-R_{S} i_{S} & ; \quad v_{b}=-R_{L} i_{1} ; \\
v_{c}=R_{1}\left(i_{1}+i_{2}\right) & ; \quad v_{d}=R_{2}\left(i_{S}-i_{2}\right) ; \\
i_{1}=g_{m_{1}}\left(v_{a}-v_{c}\right) & ; \quad i_{2}=-g_{m_{2}}\left(v_{b}-v_{d}\right) ; \\
i_{S} & =\frac{v_{a}-v_{d}}{R_{3}} ;
\end{aligned}
$$

These equations can be modified to accommodate several parasitic effects without changing the number of relevant signals. The solution to the equations is rather bulky, but proper simplifications lead to the following results:

$$
\begin{aligned}
Z_{i n} & \approx\left(1+\frac{R_{3}}{R_{2}}\right) \frac{R_{1} R_{2}}{R_{1}+R_{2}}, \\
i_{2} & \approx v_{S} \frac{1}{R_{1}\left(1+\frac{R_{S}}{Z_{i n}}\right)} .
\end{aligned}
$$

The linearity analysis is very difficult to carry out, and massive simplifications must be accepted. The approach is semi-iterative and it is based on the following assumptions:

1. the non-linearities are concentrated on the transcondutors ( $M_{1}$ and $M_{2}$ in the figure). Equation (9) is modified as follows:

$$
\begin{aligned}
& i_{1}=g_{m_{1}}\left(v_{a}-v_{c}\right)+\hat{i_{1}}, \\
& i_{2}=-g_{m_{2}}\left(v_{b}-v_{d}\right)-\hat{i_{2}},
\end{aligned}
$$

and the new system is solved with respect to nonlinear terms $\widehat{i_{1}}$ and $\widehat{i_{2}}$.

2. Third-order non-linearities are implemented through empyrical factors:

$$
\widehat{i_{1}} \triangleq \alpha_{31}\left(v_{a}-v_{c}\right)^{3}, \widehat{i_{2}} \triangleq \alpha_{32}\left(v_{b}-v_{d}\right)^{3}
$$

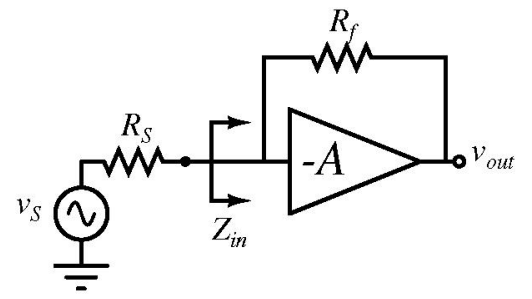

Figure 3: A simple negative-feedback stage . 


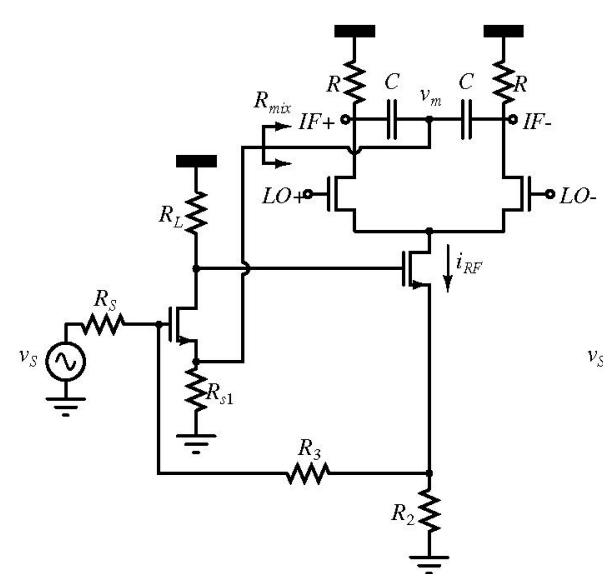

(a)

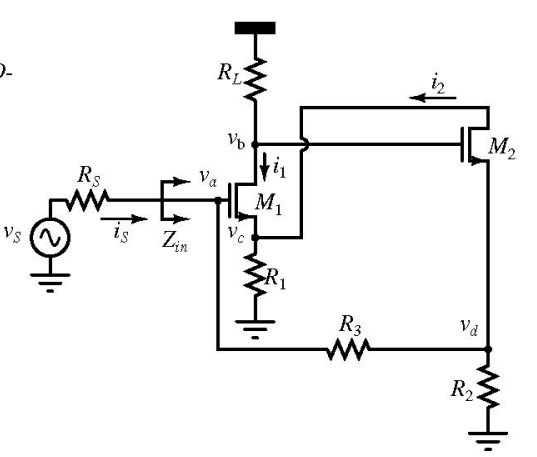

(b)

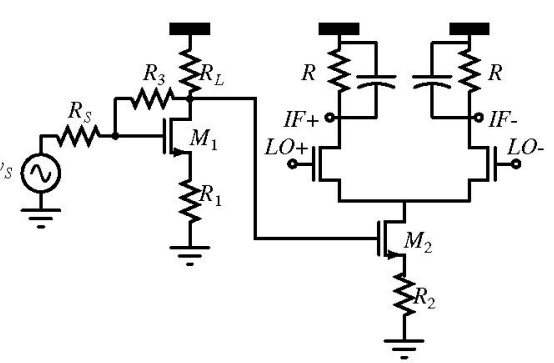

(c)

Figure 4: A dual-loop high-linearity stage: complete stage (a), simplified circuit (b) and broadband stage based on a shunt-series input amplifier (c). Biasing details are omitted .

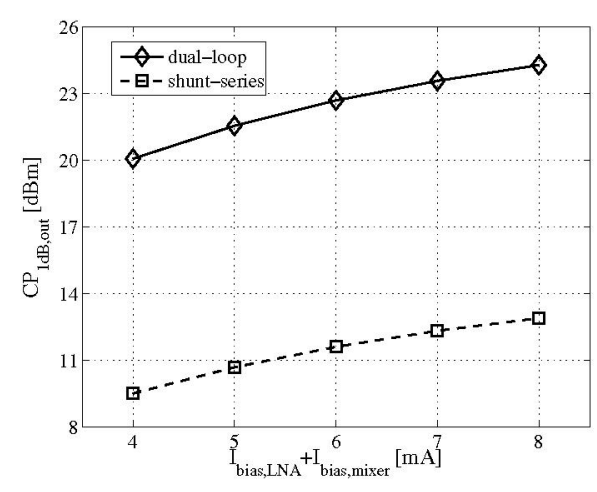

Figure 5: Output 1-dB compression point for the proposed stage and the one in Figure 4(c).

3. A polynomial equation is derived: $i_{2}=g_{s} v_{s}+\alpha_{3} v_{S}^{3}$, where $g_{s}$ is the small-signal transconductance resulting from (7)-(10), and $\alpha_{3}$ is the third-order non-linear term derived from previous equations.

As an example, we can try to compare the 1-dB compression point of the proposed stage with an RX frontend based on a shunt-series LNA and a Gilbert-cell mixer, like the one showed in Figure 4(c). Shunt-series stages are based on a local degeneration on the source (provided by $R_{1}$ ) and shunt feedback between gate and drain (see $R_{3}$ in the figure). In fact this is a dual-loop topology, but input-matching constraints limit the values of the degeneration resistance, and thereby the loop gain. To improve linearity, local feedback is applied to the mixer as well (by $R_{2}$ ). The results are shown in Figure 5: the bias current of both LNA and mixer is swept, and the output-referred $1-\mathrm{dB}$ compression point is shown. Results are based on a $0.35 \mu \mathrm{m}$-process models.

\section{Application to a narrow-band receiver}

Narrow-band LNAs based on tuned LC-tanks are implemented in many state-of-the-art receiver chains, where specifications are highly demanding. A simplified configuration is shown in Figure 6(a): the inductive degeneration provides the correct matching [4], and a cascode stage reduces the impact of the Miller effect related to Gate-Drain capacitance at transistor $M_{1}$. The mixer is built as a direct implementation of the Gilbert multiplier.

The open loop configuration can be easily modified to provide negative feedback as shown in Figure 6(b); to simplify the structure the following assumptions are made:

1. the Miller effect is negligible, the contribution of the cascode transistor in the LNA is omitted.

2. At high frequency, the load resistors of the mixer combine in parallel with the degeneration inductor $L_{s}$, and we assume that the equivalent parallel resistance of the inductor is marginally affected, and we will neglect it in the following.

3. Since we are interested in the operation around resonance, we represent the load LC tank with the equivalent parallel resistance $R_{d}$.

4. No parasitics are considered except the Gate-Source capacitance of transistor $M_{1}$. Switches and transconductors are ideal.

5. The quality factor of tuning inductor $L_{g}$ is considered infinite.

The simplified circuit results as in Figure 6(c). We can easily determine the input impedance as follows:

$$
Z_{i n}=\frac{1}{s C_{g s_{1}}}+s \cdot\left(L_{g}+L_{s}\right)+\frac{g_{m_{1}} L_{s}}{C_{g s_{1}}}\left(1+g_{m_{2}} R_{d}\right)
$$

The result does not differ from the usual one except for the multiplying factor $\left(1+g_{m_{2}} R_{d}\right)$ : given a certain unity-gain frequency $\omega_{T}=\frac{g_{m_{1}}}{C_{g s_{1}}}$, the degeneration inductor needed for proper matching will be effectively smaller.

If the design has severe current limitations, a significant term in the total noise budget will be the current noise associated with $M_{2}$, which has a spectral density equal to $4 k_{B} T \gamma g_{m_{2}}$ above the $\frac{1}{f}$-noise corner frequency. Assuming proper resonance in the input network around the operation frequency, we can calculate $M_{2}$ 's contribution 


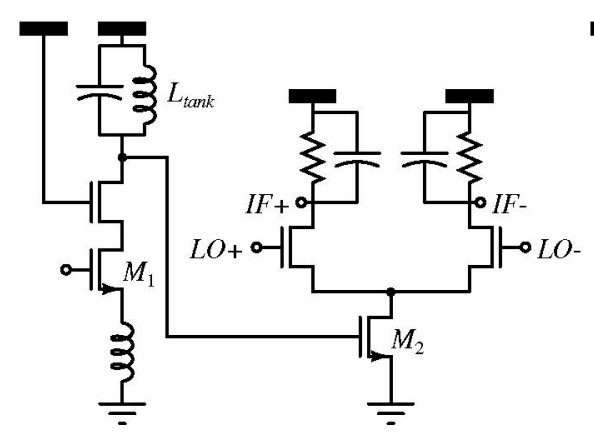

(a)

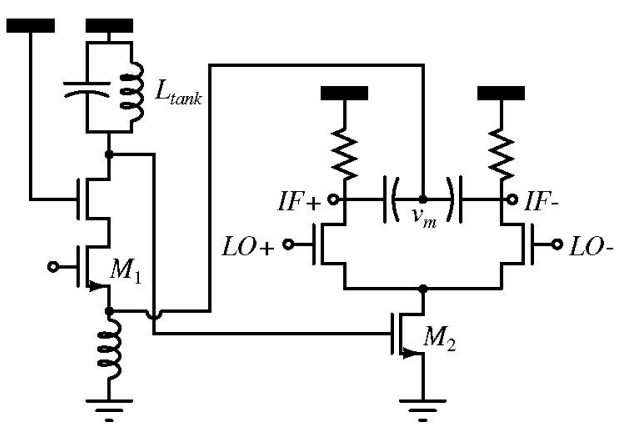

(b)

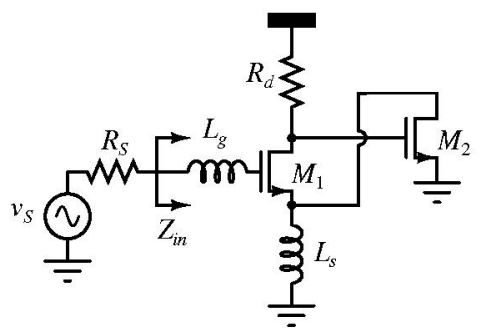

(c)

Figure 6: Evolution path leading to the proposed single-loop narrow-band receiver: typical LNA-Mixer chain (a), proposed topology (b) and simplified circuit. Biasing details are omitted.

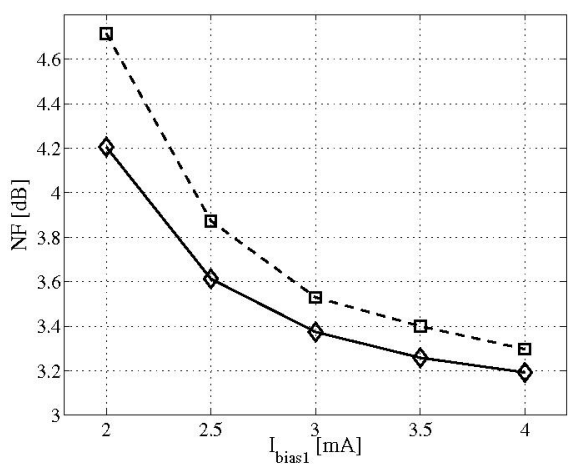

Figure 7: Comparison of NF between open-loop and closed-loop solutions.

to the total noise figure, determined by normalization with respect to the input noise spectral density $4 k_{B} T R_{S}$ :

$$
F_{M_{2}, \text { feedback }}=\frac{\left\langle i_{n, \text { out }}^{2}\right\rangle}{\left\langle i_{n, o u t_{R_{S}}}^{2}\right\rangle}=\omega^{2} \frac{\gamma g_{m_{2}} L_{s}^{2}}{R_{S}} .
$$

The same factor can be calculated for the traditional stage in Figure 6(a), under the same assumptions:

$$
\begin{aligned}
F_{M_{2}, \text { openloop }}=\frac{\left\langle i_{n, \text { out }_{M_{2}}}^{2}\right\rangle}{\left\langle i_{n, \text { out }}^{2}\right\rangle}=4 \omega^{2} \frac{\gamma g_{m_{2}} C_{g s_{1}}^{2} R_{S}}{\left(g_{m_{1}} R_{d}\right)^{2} g_{m_{2}}} \\
\frac{F_{M_{2}, \text { feedback }}}{F_{M_{2}, \text { openloop }}}=\frac{4}{\left(g_{m_{2}} R_{d}\right)^{2}} .
\end{aligned}
$$

The factor in (16) could be smaller than one, but for lowpower design the improvements might be significantly larger than unity. Figure 7 provides a comparison between a traditional open-loop solution and the proposed stage: the bias current of the input stage is swept and NF is shown. Simulations are based on a $0.35 \mu \mathrm{m}$ process, and the main parameters are summarized in Table 1.

The feedback loop does not pose serious stability problems, because in the vicinity of the resonance frequency the loop-gain magnitude can be easily determined to be smaller than unity:

$$
G_{\text {loop }}=-\frac{g_{m_{2}} R_{d}}{2+g_{m_{2}} R_{d}} .
$$

Table 1: Main parameters related to results in Figure 7.

\begin{tabular}{lccl}
\hline \hline & Closed loop & Open loop & \\
\hline$R_{S}$ & 50 & 50 & {$[\Omega]$} \\
$I_{\text {bias }_{1}}$ & $2-4$ & $2-4$ & {$[\mathrm{~mA}]$} \\
$I_{\text {bias }_{2}}$ & 2 & 2 & {$[\mathrm{~mA}]$} \\
$V_{D D}$ & 2.5 & 2.5 & {$[\mathrm{~V}]$} \\
$f_{L O}$ & 900 & 900 & {$[\mathrm{MHz}]$} \\
$\mathrm{Q} @ f_{L O}$ & 10 & 10 & \\
$L_{\text {tank }}$ & 2 & 2 & {$[\mathrm{nH}]$} \\
$f_{T}$ & $2-6$ & $2-6$ & {$[\mathrm{GHz}]$} \\
\hline \hline
\end{tabular}

\section{Conclusions}

We have devised a novel method for applying feedback techniques to traditional active mixers based on Gilbert multipliers. The approach is applied to two different topologies: a wide-band highly-reconfigurable stage exploiting dual-loop feedback, and a single-loop narrow-band frontend based on tuned LC-tank resonators. Linearity- and noise properties of the proposed circuits are analyzed and compared with more traditional solutions.

\section{References}

[1] Gilbert, B. Precise four-quadrant multiplier with subnanosecond response. IEEE Journal of Solid-State Circuits, vol. SC-3, no. 4, 1968:pp. 365-373.

[2] Lee, T. H. The design of CMOS Radio-Frequency integrated circuits (Cambridge University Press, 1998).

[3] Bruccoleri, F., Klumperink, E. and Nauta, B. Wideband CMOS low-noise amplifier exploiting thermal noise canceling. IEEE Journal of Solid-State Circuits, vol. 39, no. 2, 2004:pp. 275-282.

[4] Razavi, B. RF microelectronics (Prentice-Hall Inc., 1998). 